\title{
Adjunctive vitamin $D$ in tuberculosis treatment: meta-analysis of individual participant data
}

\author{
David A. Jolliffe (101, Davaasambuu Ganmaa², Christian Wejse ${ }^{3,4}$, \\ Rubhana Raqib ${ }^{5}$, M. Ahsanul Haq ${ }^{5}$, Nawal Salahuddin ${ }^{6}$, Peter K. Daley (10) ${ }^{7}$, Anna \\ P. Ralph ${ }^{8}$, Thomas R. Ziegler ${ }^{9}$ and Adrian R. Martineau (i) ${ }^{1}$
}

Affiliations: ${ }^{1}$ Blizard Institute, Barts and The London School of Medicine and Dentistry, Queen Mary University of London, London, UK. ${ }^{2}$ Harvard T.H. Chan School of Public Health, Boston, MA, USA. ${ }^{3}$ School of Public Health, Aarhus University, Aarhus, Denmark. ${ }^{4}$ Bandim Health Project, Statens Serum Institut, Bissau, GuineaBissau. ${ }^{5}$ International Centre for Diarrhoeal Disease Research, Dhaka, Bangladesh. ${ }^{6}$ King Faisal Specialist Hospital and Research Centre, Riyadh, Saudi Arabia. ${ }^{7}$ Memorial University, St John's, NL, Canada. ${ }^{8}$ Menzies School of Health Research, Darwin, Australia. ${ }^{9}$ Emory University School of Medicine, Atlanta, GA, USA.

Correspondence: Adrian R. Martineau, Centre for Primary Care and Public Health, Queen Mary University of London, 58 Turner Street, London, E1 2AB, UK. E-mail: a.martineaudqmul.ac.uk

@ERSpublications

A meta-analysis of individual participant data from clinical trials reports that adjunctive vitamin D accelerates sputum culture conversion in patients with multidrug-resistant tuberculosis, but not in those with drug-sensitive disease http://ow.ly/f2oN30n2OxJ

Cite this article as: Jolliffe DA, Ganmaa D, Wejse C, et al. Adjunctive vitamin D in tuberculosis treatment: meta-analysis of individual participant data. Eur Respir J 2019; 53: 1802003 [https://doi.org/10.1183/ 13993003.02003-2018].

\section{ABSTRACT}

Background: Randomised controlled trials of adjunctive vitamin D in pulmonary tuberculosis (TB) treatment have yielded conflicting results. Individual participant data meta-analysis could identify factors explaining this variation.

Methods: We meta-analysed individual participant data from randomised controlled trials of vitamin D in patients receiving antimicrobial therapy for pulmonary TB. Primary outcome was time to sputum culture conversion. Secondary outcomes were time to sputum smear conversion, mean 8-week weight and incidence of adverse events. Pre-specified subgroup analyses were done according to baseline vitamin D status, age, sex, drug susceptibility, HIV status, extent of disease and vitamin D receptor genotype.

Results: Individual participant data were obtained for 1850 participants in eight studies. Vitamin D did not influence time to sputum culture conversion overall (adjusted HR 1.06, 95\% CI 0.91-1.23), but it did accelerate sputum culture conversion in participants with multidrug-resistant pulmonary TB (adjusted HR 13.44, 95\% CI 2.96-60.90); no such effect was seen in those whose isolate was sensitive to rifampicin and/ or isoniazid (adjusted HR 1.02, 95\% CI 0.88-1.19; p-value for interaction=0.02). Vitamin D accelerated sputum smear conversion overall (adjusted HR 1.15, 95\% CI 1.01-1.31), but did not influence other secondary outcomes.

Conclusions: Vitamin D did not influence time to sputum culture conversion overall, but it accelerated sputum culture conversion in patients with multidrug-resistant pulmonary TB.

This article has supplementary material available from erj.ersjournals.com

This study is registered at PROSPERO with identifier number CRD42015020288.

Received: Oct 192018 | Accepted after revision: Dec 122018

Copyright OERS 2019 


\section{Introduction}

The World Health Organization estimates that 10 million people developed active tuberculosis (TB) in 2017 and that 1.6 million people died of this disease [1]. Existing antimicrobial treatment for active TB requires lengthy administration of a high pill burden that carries a significant risk of toxicity and has limited efficacy in drug-resistant disease. Adjunctive host-directed therapies (i.e. immunomodulatory therapies given in addition to antimicrobial treatment) have the potential to reduce duration of transmissibility, shorten antimicrobial therapy and improve outcomes in drug-resistant disease [2]. Vitamin D has attracted interest as a potential candidate on the basis of its historical use in TB treatment [3], the reported associations between vitamin D deficiency and susceptibility to TB infection and disease $[4,5]$, and its recognised role in supporting antimycobacterial immune responses [6, 7]. Double-blind, placebo-controlled randomised controlled trials (RCTs) evaluating the effects of vitamin D supplementation on sputum culture and/or smear conversion in patients receiving antimicrobial therapy for pulmonary $\mathrm{TB}$ have yielded conflicting results: four reported favourable effects on their primary outcome in the study population as a whole [8-11], two reported benefits on their primary outcome in subgroups only [12,13] and four reported no effects of the intervention on their primary outcome [14-17]. Systematic reviews and aggregate data meta-analyses including at least some of these studies [18-21] are all limited by lack of access to individual participant data, which precludes conduct of subgroup analyses to explore whether individual-level factors may modify responses to vitamin D supplementation. This is an important omission, because uneven distribution of such effect modifiers between participants in different settings might underlie variation in results seen between trials. For example, effects of vitamin D supplementation may be modified by the drug susceptibility of a patient's Mycobacterium tuberculosis isolate; this might be expected on the basis that individuals with drug-resistant disease may stand to derive particular benefit from host-directed therapy where antimicrobial therapies are less effective [2]. Alternatively, effects of vitamin D supplementation may be stronger in, or restricted to, individuals with lower baseline vitamin $\mathrm{D}$ status, since these individuals might be expected to derive the greatest benefit from vitamin $\mathrm{D}$ replacement. This phenomenon has been demonstrated in other respiratory diseases [22-25]. We therefore set out to obtain individual participant data from the 10 clinical trials [8-17] and meta-analyse them in order to obtain an updated estimate of overall efficacy, and to determine whether effects of this intervention vary according to the presence or absence of potential effect modifiers.

\section{Methods}

\section{Protocol and registration}

The methods for this systematic review and meta-analysis were described in an outline protocol that was registered with the PROSPERO International Prospective Register of Systematic Reviews (identifier CRD42015020288). Research ethics committee approval to contribute individual participant data from completed primary trials to the current meta-analysis was not required for any study. Findings are reported according to the PRISMA guidelines for individual participant data meta-analysis [26].

\section{Selection of studies and participants}

Double-blind, placebo-controlled RCTs of adjunctive vitamin D supplementation including patients receiving standard antimicrobial treatment for pulmonary $\mathrm{TB}$ were eligible for inclusion if the primary trial had been approved by a research ethics committee, and if data on sputum culture and/or smear conversion were reported. Studies in which vitamin D was given in combination with another intervention were excluded if the effects of vitamin D could not be isolated (e.g. by use of a factorial design). Studies in which a factorial design was used to investigate effects of other therapies alongside vitamin D were included, as these allowed effects of vitamin D to be isolated.

Individual participants in eligible studies were excluded if pulmonary TB was not confirmed by sputum culture or smear, i.e. we only included data from individual patients in the meta-analysis if they had either a positive sputum smear at baseline or a positive sputum culture at baseline, or both.

\section{Data sources and searches}

Two investigators (D.A.J. and A.R.M.) searched PubMed, Embase, the Cochrane Central Register of Controlled Trials (CENTRAL) and Web of Science using the electronic search strategies described in the supplementary material. Searches were regularly updated up to and including November 10, 2017. No language restrictions were imposed. Two investigators (D.A.J. and A.R.M.) determined which studies met the eligibility criteria.

\section{Data extraction and quality assurance}

Individual participant data were requested from the principal investigator for each eligible trial and the terms of collaboration were specified in a data transfer agreement, signed by representatives of the data 
provider and the recipient (Queen Mary University of London, London, UK). Data were de-identified at source prior to transfer via e-mail. On receipt, two investigators (D.A.J. and A.R.M.) assessed data integrity by performing internal consistency checks, and by cross-checking proportions of participants with positive 8-week sputum culture and smear by arm against those reported in published papers. Study authors were contacted to provide missing data and to resolve queries arising from these integrity checks. Once queries had been resolved, clean data were uploaded to the main study database, which was held in Stata/IC version 12 (StataCorp, College Station, TX, USA).

The following data relating to study characteristics were collected: study setting, eligibility criteria, details of adjunctive therapies being evaluated in each trial and duration of follow-up. Individual participant data were extracted for the following variables relating to baseline characteristics: age, sex, weight, circulating 25-hydroxyvitamin D (25(OH)D) concentration, drug susceptibility, HIV status, extent of disease on baseline chest radiograph (as measured by the proportion of zones involved and the presence or absence of cavitation), genotype for single nucleotide polymorphisms (SNPs) in the gene encoding the vitamin D receptor (VDR: FokI and TaqI), and study allocation (vitamin D versus placebo). Follow-up data were requested for time from initiation of antibiotic treatment to stable sputum culture/smear conversion (the date of stable culture/smear conversion being estimated as the mid-point between the date of the last positive sputum culture/smear and the date of the first negative sputum culture/smear thereafter); sputum culture and smear status, weight, and circulating 25(OH)D concentration after 8 weeks of antimicrobial treatment; and occurrence of serious adverse events, death, study withdrawals and potential adverse reactions to vitamin D supplementation (hypercalcaemia or renal stones).

\section{Risk of bias assessment for individual studies}

We used the Cochrane Collaboration Risk of Bias tool [27] to assess the following variables: sequence generation, allocation concealment, blinding of participants, personnel and outcome assessors, completeness of outcome data, evidence of selective outcome reporting, and other potential threats to validity. Selectivity of reporting was assessed either by comparing study protocols against study reports or by specifically asking study authors whether all pre-specified outcomes were reported. Study quality was assessed independently by two investigators (A.R.M. and D.A.J.), except for the trials in which A.R.M. was an investigator [12, 13], which were assessed by R.R. and M.A.H. Discrepancies were resolved by consensus.

\section{Definition of outcomes}

The primary outcome of the meta-analysis was time from initiation of antimicrobial therapy to stable sputum culture conversion, estimated as the mid-point between the last positive sputum culture and the first negative sputum culture thereafter. This outcome was selected as primary because it is a recognised surrogate for treatment failure and relapse that reflects the longitudinal profile of culture results over time, as opposed to a single time-point [28]. Participants who were unable to expectorate spontaneously at follow-up time-points were deemed to be culture negative. Where participants were culture positive at 8-week follow-up (or earlier if lost to follow-up before this time-point), time to sputum culture conversion was attributed as the number of days from the date of treatment initiation to the date of the last follow-up visit and the censor variable was assigned a zero value to indicate that the end-point of sputum culture conversion was not achieved. Secondary outcomes were time to stable sputum smear conversion, estimated as the mid-point between the last positive sputum smear and the first negative sputum smear thereafter; proportions of participants with negative sputum culture/smear after 8 weeks of antimicrobial therapy; weight after 8 weeks of antimicrobial therapy; and risk of death, study withdrawals and potential adverse reactions to vitamin D supplementation (hypercalcaemia or renal stones). Time to sputum culture/smear conversion was estimated only for trials where sputum was collected at intervals of $\leqslant 2$ weeks, due to the potential for imprecision of such an estimate with less frequent sputum collection.

\section{Data synthesis and analysis}

D.A.J. and A.R.M. analysed the data using Stata/IC version 12. Our individual participant data meta-analysis approach followed published guidelines [29]. We did both one-step and two-step individual participant data meta-analyses for the primary outcome of time to sputum culture conversion. For secondary outcomes, we did one-step individual participant data meta-analysis only. In the one-step approach, individual participant data from all studies were modelled simultaneously while accounting for the clustering of participants within studies. We used mixed models, with a random effect for study and fixed effects for age and sex (reflecting an assumption that the influence of age and sex on response to treatment would not vary across trials) to obtain the pooled intervention effect with a $95 \%$ confidence interval. We did not adjust for other covariates because missing values for some participants would have led to their exclusion from statistical analyses. We analysed survival data using mixed effects parametric survival models; effects of the intervention on time to sputum culture/smear conversion were expressed as 
adjusted hazard ratios. Survival data for time to sputum culture and smear conversion were truncated at 8 weeks in order to capture effects of adjunctive vitamin $\mathrm{D}$ on the standardised background of intensive-phase antimicrobial therapy. We analysed proportions of participants experiencing an event using mixed effects logistic regression; effects of the intervention on dichotomous outcomes were expressed as adjusted odds ratios. In the two-step approach, individual participant data were first analysed for each separate study independently to produce a hazard ratio for the effect of allocation on time to sputum culture conversion for that study, adjusted for age and sex. We then calculated a weighted average of adjusted hazard ratios for each study and summarised heterogeneity using the $I^{2}$ statistic.

\section{Exploration of variation in effects}

To identify factors modifying the effects of vitamin D supplementation, we did pre-specified subgroup analyses for the primary outcome of time to sputum culture conversion by extending the one-step meta-analysis framework to include treatment-covariate interaction terms. Subgroups were defined according to baseline vitamin D status (circulating $25(\mathrm{OH}) \mathrm{D}$ concentration $<25 \mathrm{versus} \geqslant 25 \mathrm{nmol} \cdot \mathrm{L}^{-1}$ ), age ( $<30$ versus $\geqslant 30$ years, where 30 years was the median value), sex, drug susceptibility (multidrug-resistant (MDR; i.e. resistant to rifampicin and isoniazid at least) versus not), HIV status (seronegative versus seropositive), extent of chest radiograph involvement at baseline $(<50 \%$ versus $\geqslant 50 \%$ of zones involved and presence versus absence of cavitation), vitamin D dosing regimen (daily/weekly versus less frequently), and VDR genotype for the FokI and TaqI SNPs (analysed using the per-allele method, i.e. under an additive model). The $25 \mathrm{nmol} \cdot \mathrm{L}^{-1}$ cut-off for baseline $25(\mathrm{OH}) \mathrm{D}$ concentration in the subgroup analyses was selected because it is the threshold for vitamin D deficiency defined by the UK Dept of Health [30] and because we have previously shown this threshold to modify host response to respiratory infection [22]. The Benjamini-Hochberg procedure for multiple testing correction was applied to the family of p-values for interaction to control the false discovery rate at $20 \%$.

\section{Risk of bias assessment across studies}

For the primary analysis, the likelihood of publication bias was investigated through the construction of a contour-enhanced funnel plot.

\section{Additional analyses}

We did a pre-specified responder analysis in participants assigned to the intervention arm of included studies, comparing time to sputum culture conversion in participants who attained a circulating $25(\mathrm{OH}) \mathrm{D}$ concentration $\geqslant 75 \mathrm{nmol} \cdot \mathrm{L}^{-1}$ versus those who did not. We did two exploratory adjustments of the subgroup analysis evaluating effects of vitamin $\mathrm{D}$ in patients with versus without MDR-TB, controlling for baseline vitamin D status $\left(25(\mathrm{OH}) \mathrm{D}<25\right.$ versus $\left.\geqslant 25 \mathrm{nmol} \cdot \mathrm{L}^{-1}\right)$ and presence versus absence of cavitation on baseline chest radiograph, in addition to the pre-specified adjustments for age, sex and clustering of participants within trials. A pre-specified sensitivity analysis excluding studies deemed at high risk of bias was not conducted, since no included trial fell into this category.

\section{Results}

Study selection and individual participant data obtained

The study selection process is illustrated in figure 1. Our search identified 513 unique studies that were assessed for eligibility, of which 10 studies with a total of 2223 randomised participants fulfilled the eligibility criteria. Individual participant data were sought for all studies and were obtained for eight out of the 10 studies (total 2096 participants). Individual participant data were not obtained for two studies (total of 127 participants): in one case the corresponding author indicated that individual participant data were not available [8]; in the other, the corresponding author did not respond to an invitation to contribute individual participant data to this meta-analysis [11]. A total of 246 randomised participants in the eight studies for which individual participant data were obtained were excluded from the meta-analysis on the basis that they did not have pulmonary TB confirmed by sputum culture and/or smear. All of the remaining 1850 participants contributed data to analysis of serious adverse events; 1163 participants contributed data to analysis of sputum culture conversion and 1611 participants contributed data to analysis of sputum smear conversion. Differences in the total number of participants contributing to these analyses represent differences in data availability: serious adverse event data were available for all, but microbiological outcome data were not.

\section{Study and participant characteristics}

The eight studies for which individual participant data were obtained were conducted in eight countries on three continents (tables 1 and 2). All studies investigated effects of vitamin $\mathrm{D}_{3}$; none investigated vitamin $\mathrm{D}_{2}$. Six studies used two-arm parallel designs to investigate effects of vitamin $\mathrm{D}_{3}$ only $[9,12-14,16,17]$; two studies investigated effects of other host-directed therapies concurrently using a factorial design [10, 15]. 


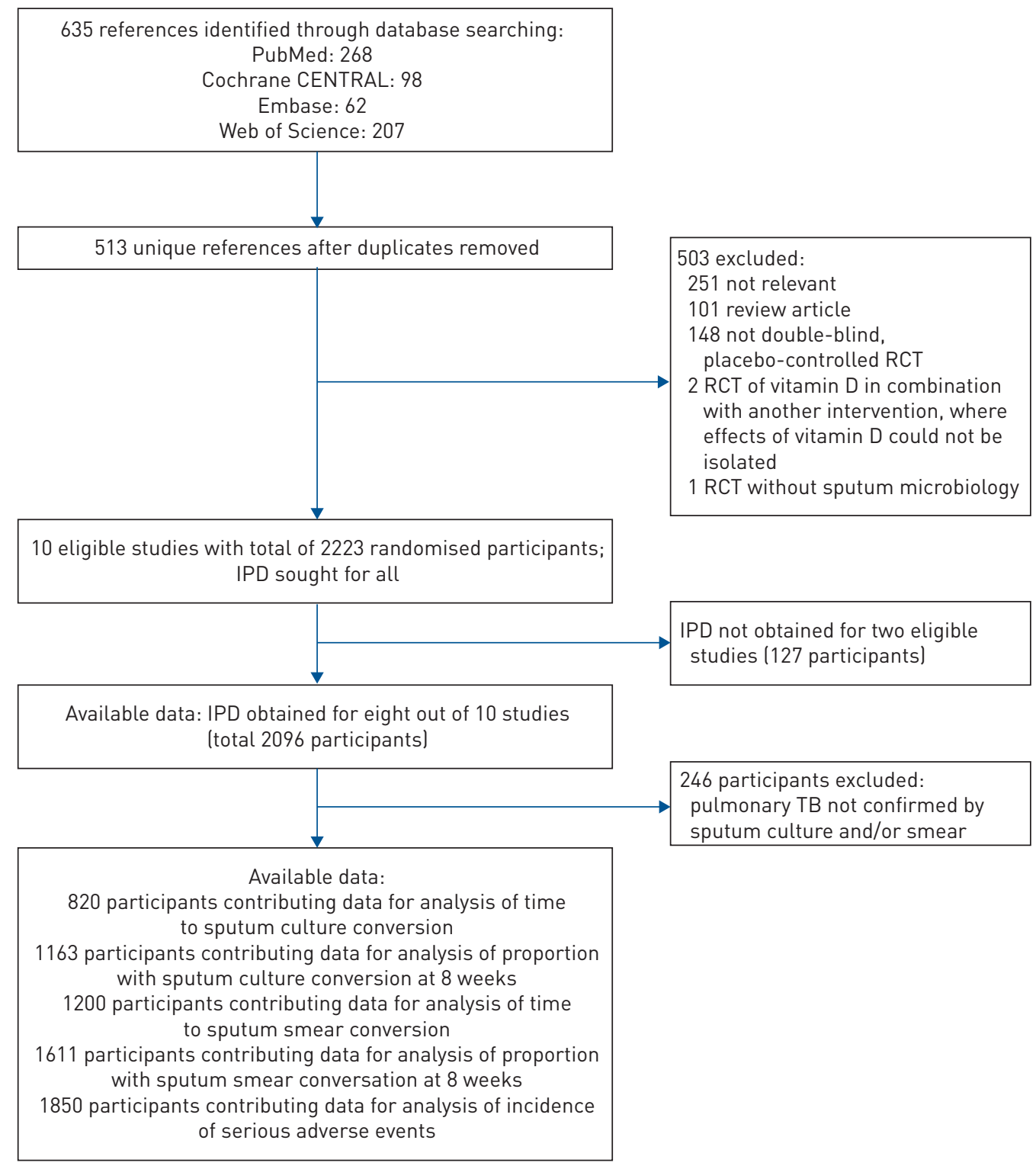

FIGURE 1 PRISMA diagram of study selection. CENTRAL: Central Register of Controlled Trials; RCT: randomised controlled trial; IPD: individual participant data; TB: tuberculosis.

Seven studies administered vitamin $\mathrm{D}_{3}$ orally to participants in the intervention arm: this was given daily [10], weekly then 2-weekly [17], 2-weekly [12, 13, 16], 4-weekly [15] and 3 times over an 8-month period (at baseline, 5 months and 8 months) [14]. One study administered vitamin $\mathrm{D}_{3}$ intramuscularly to participants in the intervention arm at baseline and 4 weeks [9]. Duration of follow-up ranged from 8 weeks to 8 months. Primary outcomes included one or more of sputum culture conversion [10,12, 13, 15-17], clinical scores $[10,14,15]$, weight gain [9] and end-study chest radiograph involvement [9]. Data relating to final treatment outcome were available for 331 participants in two trials (supplementary table S1) [14, 15]. The 1850 participants included in individual participant data meta-analysis ranged in age from 15 to 86 years and 650 (35.1\%) were female. Baseline HIV status was tested in 995 participants in five studies [12, 14-17] and found to be positive in $157(15.8 \%)$ of them. Baseline 25(OH)D concentrations were determined in 1660 participants in six studies $[10,12-14,16,17]$ and ranged from undetectable to $250 \mathrm{nmol} \cdot \mathrm{L}^{-1}: 688$ participants tested $(41.5 \%)$ had baseline $25(\mathrm{OH}) \mathrm{D}$ levels $<25 \mathrm{nmol} \cdot \mathrm{L}^{-1}$. Drug susceptibility testing results were available for 1350 participants in six studies: 55 participants tested (4.1\%) had MDR-TB (i.e. their M. tuberculosis complex isolate was resistant to isoniazid and rifampicin at least), of whom 39 (70.9\%) commenced second-line anti-TB therapy during their participation in the trials; relatively early initiation of second-line therapy was made possible by the use of molecular methods in some studies that enabled the rapid detection of mutations associated with drug resistance. Baseline characteristics of participants were 


\section{TABLE 1 Characteristics of trials and participants included in individual participant data meta-analysis: I}

\begin{tabular}{|c|c|c|c|c|c|c|c|c|c|}
\hline First author [ref.] & Setting & $\begin{array}{l}\text { Dose and route of } \\
\text { vitamin } D_{3} \text { : } \\
\text { intervention arm }\end{array}$ & Anti-TB therapy & $\begin{array}{l}\text { Included/ } \\
\text { randomised }\end{array}$ & $\begin{array}{l}\text { Allocated to } \\
\text { vitamin D/ } \\
\text { placebo }\end{array}$ & $\begin{array}{l}\text { Age } \\
\text { years }\end{array}$ & $\begin{array}{c}\text { Sex ratio } \\
M / F\end{array}$ & $\begin{array}{c}\text { Proportion HIV } \\
\text { infected at } \\
\text { baseline }^{\#}\end{array}$ & $\begin{array}{l}\text { Proportion with } \\
\text { MDR-TB }\end{array}$ \\
\hline WEJSE 2009 [14] & Guinea-Bissau & $\begin{array}{c}2.5 \mathrm{mg} \text { at 0/5/8 months, } \\
\text { oral }\end{array}$ & $\begin{array}{c}2 \text { months HRZE, then } \\
6 \text { months HE }\end{array}$ & $241 / 367^{\uparrow}$ & $117 / 124$ & $\begin{array}{c}36.1 \pm 13.4 \\
(16-80)\end{array}$ & $144 / 97$ & $\begin{array}{l}82 / 240 \\
(34.2)\end{array}$ & ND \\
\hline Martineau 2011 [12] & UK & $\begin{array}{c}2.5 \mathrm{mg} \text { at } 0 / 2 / 4 / 6 \text { weeks, } \\
\text { oral }\end{array}$ & 2 months HRZE & $135 / 146^{+}$ & $70 / 65$ & $\begin{array}{c}33.4 \pm 11.6 \\
(18-75)\end{array}$ & $103 / 32$ & $\begin{array}{l}5 / 93 \\
(5.4)\end{array}$ & $\begin{array}{l}1 / 135 \\
(0.7)\end{array}$ \\
\hline SaLAHUdDIN 2013 [9] & Pakistan & $15 \mathrm{mg}$ at $0 / 4$ weeks, i.m. & $\begin{array}{c}2 \text { months HRZE, then } \\
6 \text { months HE }\end{array}$ & $259 / 259$ & $132 / 127$ & $\begin{array}{c}28.1 \pm 13.6 \\
(16-86)\end{array}$ & $141 / 118$ & ND & ND \\
\hline RaLPH 2013 [15] & Indonesia & $\begin{array}{c}1.25 \mathrm{mg} \text { at } 0 / 4 \text { weeks, oral } \\
\text { (factorial design with } \\
\text { L-arginine) }\end{array}$ & $\begin{array}{c}2 \text { months HRZE then } \\
4 \text { months HR }\end{array}$ & $164 / 200^{\S}$ & $86 / 78$ & $\begin{array}{c}31.0 \pm 11.2 \\
(15-65)\end{array}$ & $109 / 55$ & $\begin{array}{l}19 / 123 \\
(15.4)\end{array}$ & $\begin{array}{l}2 / 164 \\
(1.2)\end{array}$ \\
\hline MıLY 2015 [10] & Bangladesh & $\begin{array}{l}0.125 \mathrm{mg} \text { daily for } \\
2 \text { months, oral } \\
\text { (factorial design with oral } \\
\text { 4-phenylbutyrate) }\end{array}$ & $\begin{array}{c}2 \text { months HRZE then } \\
4 \text { months HR }\end{array}$ & $260 / 288^{f}$ & $132 / 128$ & $\begin{array}{l}27.2 \pm 8.0 \\
(18-59)\end{array}$ & $162 / 98$ & ND & $\begin{array}{l}7 / 260 \\
(2.7)\end{array}$ \\
\hline DaLey 2015 [16] & India & $\begin{array}{c}2.5 \mathrm{mg} \text { at } 0 / 2 / 4 / 6 \text { weeks, } \\
\text { oral }\end{array}$ & 2 months HRZE & $209 / 247^{\# \#}$ & $101 / 108$ & $\begin{array}{c}43.0 \pm 14.8 \\
(18-72)\end{array}$ & $162 / 47$ & $\begin{array}{l}0 / 209 \\
(0)\end{array}$ & $\begin{array}{l}1 / 209 \\
(0.5)\end{array}$ \\
\hline Tukvadze 2015 [17] & $\begin{array}{l}\text { Republic of } \\
\text { Georgia }\end{array}$ & $\begin{array}{c}1.25 \mathrm{mg} \text { weekly for } 8 \text { weeks, } \\
\text { then alternate weeks for } \\
8 \text { weeks, oral }\end{array}$ & $\begin{array}{c}2 \text { months HRZE then } \\
4 \text { months HR }\end{array}$ & 192/199ๆา & $97 / 95$ & $\begin{array}{c}33.5 \pm 11.6 \\
(18-63)\end{array}$ & $123 / 69$ & $\begin{array}{l}3 / 184 \\
(1.6)\end{array}$ & $\begin{array}{l}23 / 192 \\
(12.0)\end{array}$ \\
\hline GanmaA 2017 [13] & Mongolia & $\begin{array}{c}3.5 \mathrm{mg} \text { at } 0 / 2 / 4 / 6 \text { weeks, } \\
\text { oral }\end{array}$ & 2 months HRZE & $390 / 390$ & $190 / 200$ & $\begin{array}{c}35.9 \pm 13.9 \\
(17-84)\end{array}$ & $256 / 134$ & ND & $\begin{array}{c}21 / 390 \\
(5.4)\end{array}$ \\
\hline
\end{tabular}

Data are presented as $n$, mean \pm SD (range) or $n / N(\%)$, unless otherwise stated. TB: tuberculosis; H: isoniazid; R: rifampicin; Z: pyrazinamide; E: ethambutol; M: male; F: female; ND: not determined; MDR: multidrug-resistant (i.e. resistant to $\mathrm{H}$ and $\mathrm{R}$ at least). ${ }^{\#}$ : where denominators are less than total number of included participants, data are missing; ${ }^{\natural}: 126$ excluded (113 smear negative at baseline, 11 extrapulmonary TB only, two with final diagnosis other than TB); ${ }^{+}: 11$ excluded (eight cultured nontuberculous mycobacteria, three culture negative at baseline); ${ }^{\S}: 36$ excluded (19 culture negative at baseline, 13 culture missing at baseline, four culture contaminated at baseline); ${ }^{f}: 28$ excluded (all culture negative at baseline); ${ }^{\# \#: ~} 38$

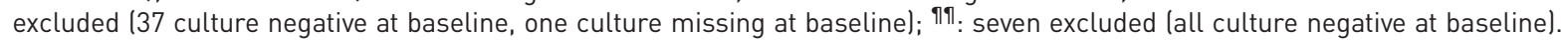




\begin{tabular}{|c|c|c|c|c|c|c|c|}
\hline First author [ref.] & $25(\mathrm{OH}) \mathrm{D}$ assay & $\begin{array}{c}\text { Baseline } 25(\mathrm{OH}) \mathrm{D}^{\#} \\
\mathrm{nmol} \cdot \mathrm{L}^{-1}\end{array}$ & $\begin{array}{l}\text { Proportion with baseline } \\
25(\mathrm{OH}) \mathrm{D}<25 \mathrm{nmol} \cdot \mathrm{L}^{-1}\end{array}$ & $\begin{array}{l}\text { Duration of } \\
\text { follow-up }\end{array}$ & Primary outcome & $\begin{array}{l}\text { Sputum culture } \\
\text { ascertainment }\end{array}$ & $\begin{array}{l}\text { Sputum smear } \\
\text { ascertainment }\end{array}$ \\
\hline WEJSE 2009 [14] & LC-MS/MS & $\begin{array}{c}77.7 \pm 23.2 \\
(35-191)\end{array}$ & $\begin{array}{c}0 / 232 \\
(0.0)\end{array}$ & 8 months & Clinical score & ND & $\begin{array}{c}0,2,4,6,8 \text { weeks, } 5 \\
8 \text { months }\end{array}$ \\
\hline MaRtineAu 2011 [12] & LC-MS/MS & $\begin{array}{l}20.7 \pm 19.1 \\
(U D-94)\end{array}$ & $\begin{array}{l}96 / 135 \\
(71.1)\end{array}$ & 8 weeks & Sputum culture conversion & $0,2,4,6,8$ weeks & $0,2,4,6,8$ weeks \\
\hline SALAHUDDIN 2013 [9] & ECLIA & $\begin{array}{c}54.2 \pm 23.7 \\
(10-135)\end{array}$ & $\begin{array}{c}36 / 259 \\
(13.9]\end{array}$ & 12 weeks & $\begin{array}{l}\text { Weight gain and chest } \\
\text { radiograph involvement } \\
\text { (co-primary) }\end{array}$ & ND & $0,4,8,12$ weeks \\
\hline RALPH 2013 [15] & NA & ND & ND & 24 weeks & $\begin{array}{l}\text { Sputum culture conversion } \\
\text { and clinical score } \\
\text { (co-primary) }\end{array}$ & $0,4,8$ weeks & $\begin{array}{c}0,1,2,3,4,5,6,7,8,12, \\
16,20,24 \text { weeks }\end{array}$ \\
\hline MıLY 2015 [10] & ECLIA & $\begin{array}{c}26.7 \pm 16.3 \\
(8-91)\end{array}$ & $\begin{array}{c}142 / 256 \\
(55.5)\end{array}$ & 24 weeks & $\begin{array}{l}\text { Sputum culture conversion } \\
\text { and clinical score } \\
\text { (co-primary) }\end{array}$ & $0,4,8$ weeks & $\begin{array}{c}0,1,2,3,4,6,8,10,12 \\
24 \text { weeks }\end{array}$ \\
\hline Daley 2015 [16] & ECLIA & $\begin{array}{l}63.0 \pm 49.7 \\
(10-250)\end{array}$ & $\begin{array}{c}34 / 204 \\
(16.7)\end{array}$ & 8 weeks & Sputum culture conversion & $0,2,4,6,8$ weeks & $0,2,4,6,8$ weeks \\
\hline Tukvadze 2015 [17] & LC-MS/MS & $\begin{array}{c}37.4 \pm 22.1 \\
(6-104)\end{array}$ & $\begin{array}{l}69 / 190 \\
(36.3)\end{array}$ & 16 weeks & Sputum culture conversion & $\begin{array}{c}0,2,4,6,8,12 \\
16 \text { weeks }\end{array}$ & 0,8 weeks \\
\hline GanmaA 2017 [13] & CLIA & $\begin{array}{l}17.2 \pm 24.2 \\
\text { (UD-188) }\end{array}$ & $\begin{array}{c}311 / 384 \\
(81.0)\end{array}$ & 8 weeks & Sputum culture conversion & $0,2,4,6,8$ weeks & $0,2,4,6,8$ weeks \\
\hline
\end{tabular}




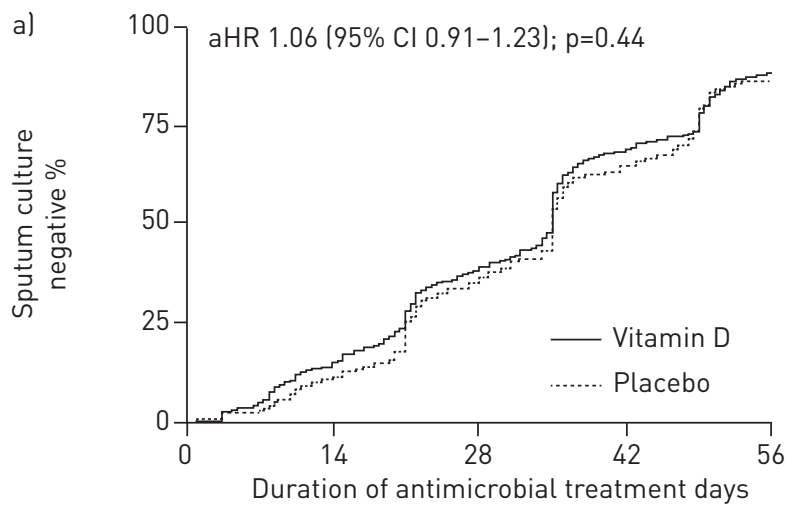

At risk $\mathrm{n}$

Vitamin D

Placebo

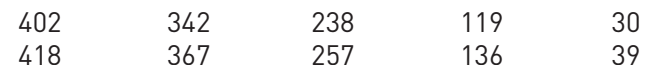
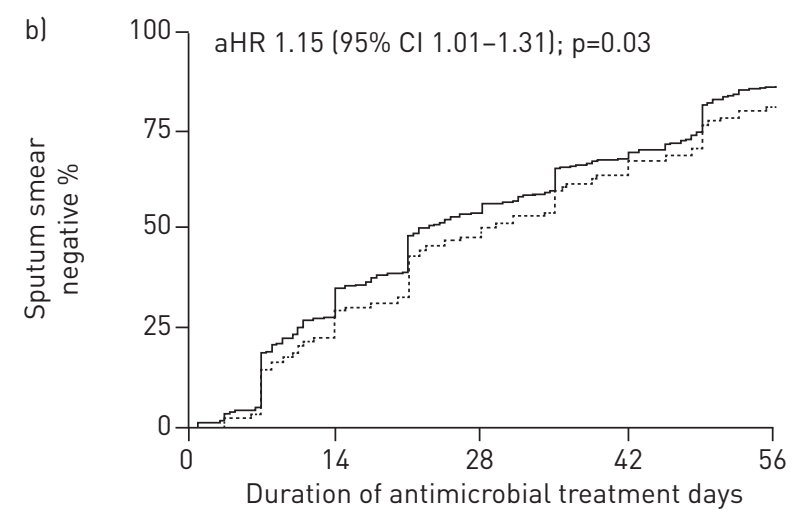

At risk $n$

Vitamin D

Placebo

\begin{tabular}{|c|c|c|c|}
\hline 598 & 420 & 260 & 175 \\
\hline 602 & 459 & 298 & 201 \\
\hline
\end{tabular}

FIGURE 2 a) Time to sputum culture conversion and b) time to sputum smear conversion by allocation: one-step individual participant data meta-analysis. Numbers of participants with positive sputum culture or smear remaining in follow-up (number at risk) at $0,14,28,42$ and 56 days are shown. Values for adjusted hazard ratios (aHRs) and $95 \%$ confidence intervals are from mixed models with a random effect for study and fixed effects for age and sex.

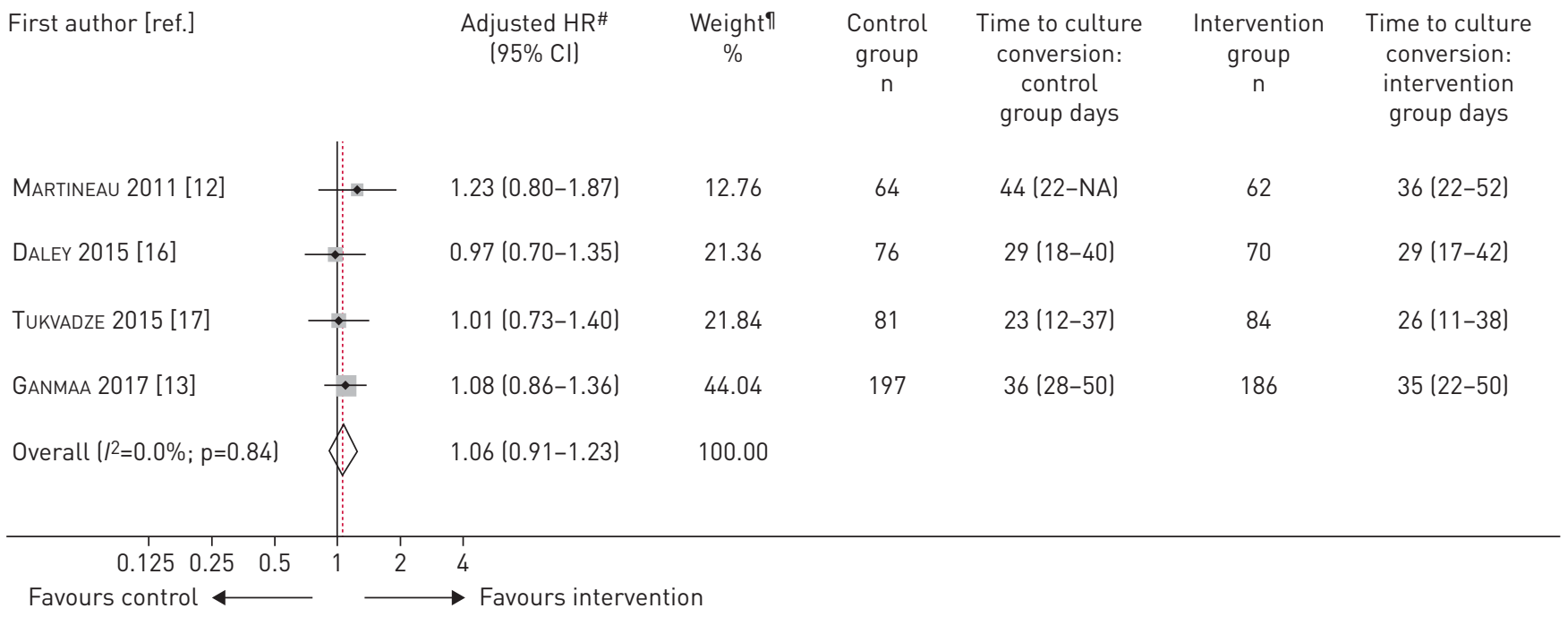

FIGURE 3 Forest plot from two-step individual participant data meta-analysis of adjusted hazard ratios: time to sputum culture conversion by allocation to control (placebo) or intervention (vitamin D). NA: not available, since 75th centiles for time to culture conversion in this group cannot be defined. Data for time to culture conversion are presented as median (interquartile range). " : adjusted for age and sex; " from random effects analysis. 
TABLE 3 One-step individual participant data meta-analysis: time to sputum culture conversion after initiation of antimicrobial therapy (overall and by subgroup)

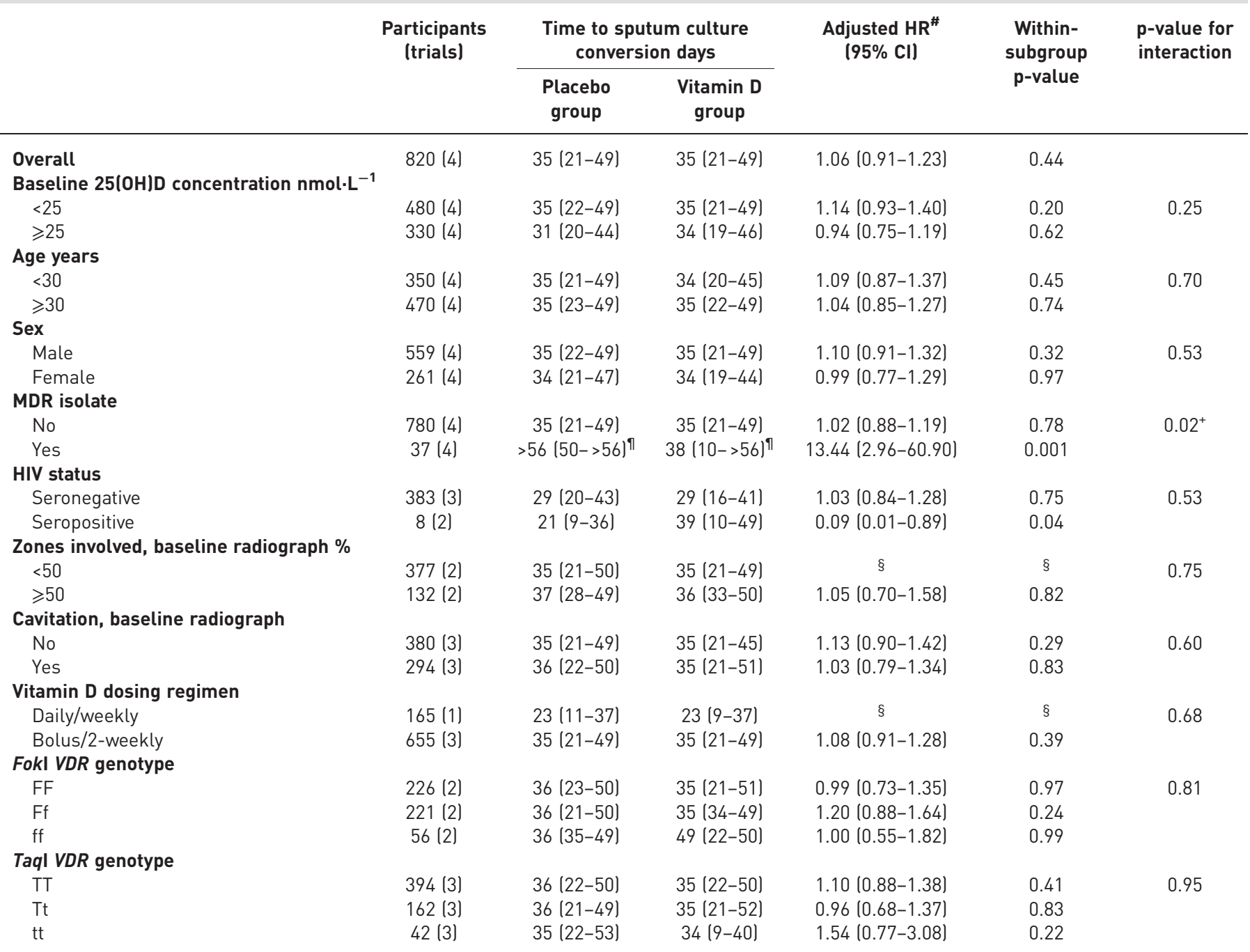

Data are presented as $\mathrm{n}$ or median (interquartile range), unless otherwise stated. 25(OH)D: 25-hydroxyvitamin D; MDR: multidrug-resistant (i.e. resistant to isoniazid and rifampicin at least); VDR: vitamin D receptor. "\#: adjusted for age, sex and clustering within trials, except for subgroup analysis by age (where age was not adjusted for) and sex (where sex was not adjusted for); ": survival times were truncated at 56 days in order to capture effects of adjunctive vitamin $\mathrm{D}$ on the standardised background of intensive-phase antimicrobial therapy; values for time to sputum culture conversion of $>56$ days are therefore indicated as " $>56 " ;{ }^{+}$: $p$-value for interaction for this subgroup analysis remained significant after correction for multiple comparisons testing, using the Benjamini-Hochberg method with a false discovery rate of $20 \%$; ${ }^{\S}$ : within-subgroup adjusted hazard ratio and $p$-value could not be calculated due to nonconvergence of the model.

(adjusted HR 1.06, 95\% CI 0.91-1.23; $\mathrm{p}=0.44 ; 820$ participants in four studies $[12,13,16,17]$ ) (figure 2a) or in two-step analysis (adjusted HR 1.06, 95\% CI 0.91-1.23; p=0.46, p-value for heterogeneity $=0.84 ; 820$ participants in four studies $[12,13,16,17]$ ) (figure 3 ).

\section{Subgroup analyses: primary outcome}

Subgroup analyses were conducted using one-step individual participant data meta-analysis to investigate whether the effects of adjunctive vitamin D supplementation on time to sputum culture conversion differed according to baseline vitamin D status, age, sex, drug susceptibility, HIV status, extent of disease on baseline chest radiograph, presence or absence of cavitation on baseline chest radiograph, type of dosing regimen and VDR genotype. Results are presented in table 3. Vitamin D supplementation accelerated sputum culture conversion among individuals with MDR pulmonary TB (adjusted HR 13.44, 95\% CI 2.96-60.90; within-subgroup $\mathrm{p}=0.001 ; 37$ participants in four studies $[12,13,16,17])$, but not in those whose isolate was sensitive to rifampicin and/or isoniazid (adjusted HR 1.02, 95\% CI 0.88-1.19; 
within-subgroup $\mathrm{p}=0.78, \mathrm{p}$-value for interaction $=0.02 ; 780$ participants in four studies $[12,13,16,17]$ ). The p-value for interaction for this subgroup analysis remained significant after correction for multiple comparisons testing, using the Benjamini-Hochberg method with a false discovery rate of $20 \%$. Because we noted a degree of imbalance in the proportion of patients with MDR-TB who had cavitation in intervention versus control arms (26.7\% versus $48.0 \%$, respectively; supplementary table S3) we conducted an exploratory subgroup analysis additionally controlling for presence versus absence of cavitation. Results of the subgroup analysis were not materially affected (within-subgroup adjusted HR for patients with MDR-TB 11.43, 95\% CI 2.22-58.94; within-subgroup $\mathrm{p}=0.004$, $\mathrm{p}$-value for interaction=0.03; 36 participants in three studies $[12,13,17])$. We also noted that the prevalence of baseline vitamin D deficiency was higher in participants with versus without MDR-TB (61.8\% versus $41.4 \%$, respectively; $\mathrm{p}=0.003$ ). However, results of the subgroup analysis were not materially affected when additional adjustment was made for the presence versus the absence of vitamin $\mathrm{D}$ deficiency at baseline ( $\mathrm{p}$-value for interaction $=0.02$ ). $\mathrm{p}$-values for interaction for all other subgroup analyses were $>0.05$.

\section{Secondary outcomes: efficacy}

Adjunctive vitamin D accelerated sputum smear conversion (median time to sputum smear conversion 21 days for vitamin D versus 26 days for placebo: adjusted HR 1.15, 95\% CI 1.01-1.31; p=0.03; 1200 participants in six studies $[10,12-16]$ ) (figure 2b). However, the proportion of participants with negative sputum culture and smear at 8 weeks was not significantly different for those randomised to vitamin D versus placebo (490 out of 577 (84.9\%) versus 484 out of $586(82.6 \%)$ culture negative at 8 weeks: adjusted OR 1.17, 95\% CI 0.85-1.60; p=0.33; 1163 participants in six studies [10, 12, 13, 15-17] and 644 out of 806 (79.9\%) versus 608 out of 805 (75.5\%) smear negative at 8 weeks: adjusted OR 1.29, 95\% CI 1.00-1.67; $\mathrm{p}=0.051 ; 1611$ participants in eight studies $[9,10,12-17])$. Allocation did not influence mean weight after 8 weeks of antimicrobial therapy in participants randomised to vitamin D versus placebo (53.2 versus $53.4 \mathrm{~kg}$, respectively: mean difference $0.13 \mathrm{~kg}, 95 \% \mathrm{CI}-0.24-0.49 \mathrm{~kg} ; \mathrm{p}=0.43 ; 1634$ participants in eight studies $[9,10,12-17])$.

\section{Secondary outcomes: safety}

Results of one-step individual participant data meta-analysis of safety outcomes are reported in supplementary table S5. No participant experienced renal stones. There was no difference in the proportion of participants allocated to vitamin D versus placebo experiencing hypercalcaemia (6.2\% versus $6.1 \% ; \mathrm{p}=0.83)$, serious adverse events of any cause $(3.6 \%$ versus $3.5 \% ; \mathrm{p}=0.96)$, study withdrawal $(12.6 \%$ versus $13.4 \%$; $\mathrm{p}=0.79)$ or death due to any cause $(2.5 \%$ versus $2.3 \% ; \mathrm{p}=0.79)$.

\section{Risk of bias across studies}

A funnel plot for the outcome of time to sputum culture conversion did not suggest publication bias in relation to this outcome, since RCTs showed an even spread of results on both sides of the overall adjusted hazard ratio (supplementary figure $\mathrm{S} 1$ ).

\section{Responder analysis}

Results of responder analysis are presented in supplementary table S6. Among participants randomised to the intervention arm of studies for which end-study $25(\mathrm{OH}) \mathrm{D}$ data were available, no difference in time to sputum culture conversion was observed between participants who attained serum $25(\mathrm{OH}) \mathrm{D}$ $\geqslant 75 \mathrm{nmol} \cdot \mathrm{L}^{-1}$ versus those who did not.

\section{Discussion}

We report results of the first meta-analysis of individual participant data from RCTs of adjunctive vitamin $\mathrm{D}$ in patients with pulmonary TB. In the study population as a whole, vitamin D supplementation did not influence the primary outcome of time to sputum culture conversion, but it did modestly accelerate sputum smear conversion, which was a secondary outcome. Pre-specified subgroup analysis revealed that vitamin D accelerated sputum culture conversion in participants with MDR-TB, but not in those whose mycobacterial isolate was sensitive to rifampicin or isoniazid, or both. Vitamin D supplementation was safe at the doses administered: no instances of renal stones were seen and serious adverse events were evenly distributed between participants randomised to vitamin D versus placebo.

Our overall finding of no effect of vitamin D on time to sputum culture conversion is consistent with results from existing systematic reviews and aggregate data meta-analyses, although our findings of accelerated sputum smear conversion are at variance with them [18-21]. In contrast to the lack of effect overall, the favourable effect of vitamin $\mathrm{D}$ on time to culture conversion that we demonstrate in the subgroup of patients with MDR-TB is of potential clinical significance. Moreover, it is biologically plausible, since host-directed therapies such as vitamin D are likely to confer greater benefit in scenarios 
where antimicrobial therapy is less effective [2]. Caution in interpreting results of this subgroup analysis is warranted for several reasons. First, a significant minority $(29.1 \%)$ of participants with MDR-TB did not receive any second-line antimicrobial therapy during their participation in the trials and therefore findings cannot be generalised to patients receiving optimal antimicrobial treatment for MDR-TB. Second, the analysis is based on a relatively small number of participants; accordingly, the $95 \%$ confidence interval for the adjusted hazard ratio is wide. Third, the potential for a positive result arising by chance as a consequence of several subgroup analyses being conducted cannot be discounted. However, we minimised the potential for type 1 error by pre-specifying a limited number of subgroup analyses, each of which was supported by an independent hypothesis; the p-value for interaction for this subgroup analysis (0.02) remained significant after correction for multiple comparisons testing. All in all, when taken together with data from observational studies showing that low $25(\mathrm{OH}) \mathrm{D}$ associates with delayed sputum smear conversion in patients receiving second-line therapy for MDR pulmonary TB [31], and considering the low cost and low toxicity of adjunctive vitamin D supplementation, the positive finding in this subgroup provides a rationale for conducting a new RCT to investigate the effects of this intervention in patients receiving second-line antimicrobial therapy for MDR-TB. However, in our judgement, the strength of evidence from this subgroup analysis is not sufficient to justify a clinical recommendation to use adjunctive vitamin $\mathrm{D}$ in the treatment of MDR-TB without such a new primary trial being conducted.

\section{Strengths and limitations of this study}

Our study has several strengths. It is unique in that we had access to individual participant data: this allowed us to conduct subgroup analyses to determine whether effects of vitamin $\mathrm{D}$ supplementation varied between individuals. We included data from eight out of 10 eligible studies $(88 \%$ of participants worldwide), none of which were assessed as being at high risk of bias, and restricted analysis to patients who had microbiologically confirmed TB. Included studies recruited patients from low- and high-incidence settings in three continents, enhancing the generalisability of our results. Our primary outcome of time to sputum culture conversion is accepted as the preferred end-point for phase 2 trials of new TB regimens [28]. Our definition captured stable culture conversion only, i.e. if a patient became culture negative but subsequently reverted to culture positive, this was not classified as sputum culture conversion.

Our study also has some limitations. We failed to obtain individual participant data from two out of the 10 eligible trials $[8,11]$. However, neither of these studies investigated our primary outcome of sputum culture conversion and both were small ( $n=67$ and $n=60)$; their omission therefore has a limited impact on secondary outcomes only. Of note, both trials reported favourable effects of vitamin $\mathrm{D}$ on their primary outcome; therefore, if their omission does introduce a bias, that bias would likely be towards the null. Interpretation of the funnel plot (supplementary figure S1) is limited by the small number of studies included, but the fact that the smaller RCTs showed an equal spread of results on both sides of the overall adjusted hazard ratio provides some reassurance that publication bias was not a major issue in our meta-analysis. A further limitation is that end-of-treatment outcomes were not available for meta-analysis: this reflects the fact that almost all trials had relatively short follow-up. However, in the absence of a consistent signal from the phase $2 \mathrm{~B}$ studies included in this meta-analysis, phase 3 trials of adjunctive vitamin $\mathrm{D}$ with end-of-treatment outcomes are not likely to be conducted. Our meta-analysis therefore represents the best evidence in this field that is likely to become available.

\section{Conclusions and policy implications}

In conclusion, we show that adjunctive vitamin $\mathrm{D}$ does not influence time to sputum culture conversion in drug-sensitive pulmonary $\mathrm{TB}$, but it may accelerate sputum culture conversion in patients with MDR disease. RCTs of vitamin D supplementation in patients with MDR pulmonary TB are therefore justified.

Acknowledgements: We thank all the people who participated in primary RCTs and the teams who conducted them. We also thank Richard Hooper and Lauren Greenberg (Queen Mary University of London, London, UK) for helpful advice regarding statistical analyses.

Transparency declaration: A.R. Martineau is the manuscript's guarantor, and he affirms that the manuscript is an honest, accurate and transparent account of the study being reported. The study was conducted as pre-specified in the protocol, except for the following aspects. Subgroup analysis of the primary outcome by vitamin D dose $(<4000$ versus $\geqslant 4000 \mathrm{IU}$ day $^{-1}$ or equivalent) was not performed, since studies investigating doses of $<4000 \mathrm{IU} \cdot \mathrm{day}^{-1}$ or equivalent [14, 15] did not evaluate time to sputum culture conversion. Subgroup analysis of the primary outcome by type of vitamin $\mathrm{D}$ administered $\left(\mathrm{D}_{2}\right.$ versus $\left.\mathrm{D}_{3}\right)$ was not performed, since vitamin $\mathrm{D}_{2}$ was not administered in any included study: all investigated vitamin $\mathrm{D}_{3}$. A pre-specified sensitivity analysis excluding studies deemed at high risk of bias was not conducted, since no included trial fell into this category. In response to reviewers' requests we did two exploratory adjustments of the subgroup analysis evaluating effects of vitamin D in patients with versus without MDR-TB in addition to the pre-specified adjustments for age, sex and clustering of participants within trials: one included additional adjustment for baseline vitamin D status $\left(25(\mathrm{OH}) \mathrm{D}<25\right.$ versus $\left.\geqslant 25 \mathrm{nmol} \cdot \mathrm{L}^{-1}\right)$ and the other included additional adjustment for presence versus absence of cavitation on baseline chest radiograph. 
Author contributions: A.R. Martineau initiated the project and wrote the study protocol. D.A. Jolliffe and A.R. Martineau assessed eligibility of studies for inclusion. D.A. Jolliffe, A.R. Martineau, R. Raqib and M.A. Haq performed risk of bias assessments. D. Ganmaa, C. Wejse, R. Raqib, N. Salahuddin, P.K. Daley, A.P. Ralph, T.R. Ziegler and A.R. Martineau were all directly involved in the acquisition and supply of individual participant data for the work. D.A. Jolliffe designed and executed statistical analyses, with input from A.R. Martineau. A.R. Martineau and D.A. Jolliffe wrote the first draft of the report. All authors revised it critically for important intellectual content, gave final approval of the version to be published, and agreed to be accountable for all aspects of the work in ensuring that questions relating to the accuracy or integrity of any part of the work were appropriately investigated and resolved.

Conflict of interest: D.A. Jolliffe has nothing to disclose. D. Ganmaa has nothing to disclose. C. Wejse has nothing to disclose. R. Raqib has nothing to disclose. M.A. Haq has nothing to disclose. N. Salahuddin has nothing to disclose. P.K. Daley has nothing to disclose. A.P. Ralph reports grants from Australian National Health and Medical Research Council, during the conduct of the study; one of the trials included in this meta-analysis was funded by the Australian National Health and Medical Research Council. T.R. Ziegler has nothing to disclose. A.R. Martineau has nothing to disclose.

Support statement: A.R. Martineau and D.A. Jolliffe are supported by the Higher Education Funding Council for England (HEFCE)

\section{References}

World Health Organization. Global Tuberculosis Report 2018. Geneva, WHO, 2018.

Wallis RS, Hafner R. Advancing host-directed therapy for tuberculosis. Nat Rev Immunol 2015; 15: 255-263. Martineau AR, Honecker FU, Wilkinson RJ, et al. Vitamin D in the treatment of pulmonary tuberculosis. J Steroid Biochem Mol Biol 2007; 103: 793-798.

4 Ganmaa D, Khudyakov P, Buyanjargal U, et al. Prevalence and determinants of QuantiFERON-diagnosed tuberculosis infection in 9,810 Mongolian schoolchildren. Clin Infect Dis 2018; in press [https://doi.org/10.1093/cid/ciy975].

5 Martineau AR, Nhamoyebonde S, Oni T, et al. Reciprocal seasonal variation in vitamin D status and tuberculosis notifications in Cape Town, South Africa. Proc Natl Acad Sci USA 2011; 108: 19013-19017.

6 Liu PT, Stenger S, Li H, et al. Toll-like receptor triggering of a vitamin D-mediated human antimicrobial response. Science 2006; 311: 1770-1773.

7 Martineau AR, Wilkinson KA, Newton SM, et al. IFN-gamma- and TNF-independent vitamin D-inducible human suppression of mycobacteria: the role of cathelicidin LL-37. J Immunol 2007; 178: 7190-7198.

8 Nursyam EW, Amin Z, Rumende CM. The effect of vitamin D as supplementary treatment in patients with moderately advanced pulmonary tuberculous lesion. Acta Med Indones 2006; 38: 3-5.

9 Salahuddin N, Ali F, Hasan Z, et al. Vitamin D accelerates clinical recovery from tuberculosis: results of the SUCCINCT Study [Supplementary Cholecalciferol in recovery from tuberculosis]. A randomized, placebo-controlled, clinical trial of vitamin D supplementation in patients with pulmonary tuberculosis'. BMC Infect Dis 2013; 13: 22.

10 Mily A, Rekha RS, Kamal SM, et al. Significant effects of oral phenylbutyrate and vitamin D3 adjunctive therapy in pulmonary tuberculosis: a randomized controlled trial. PLoS One 2015; 10: e0138340.

11 Farazi A, Didgar F, Sarafraz A. The effect of vitamin D on clinical outcomes in tuberculosis. Egypt J Chest Dis Tuberc 2017; 66: 419-423.

12 Martineau AR, Timms PM, Bothamley GH, et al. High-dose vitamin $\mathrm{D}_{3}$ during intensive-phase antimicrobial treatment of pulmonary tuberculosis: a double-blind randomised controlled trial. Lancet 2011; 377: 242-250.

13 Ganmaa D, Munkhzul B, Fawzi W, et al. High-dose vitamin $\mathrm{D}_{3}$ during tuberculosis treatment in Mongolia: a randomised controlled trial. Am J Respir Crit Care Med 2017; 195: 628-637.

14 Wejse C, Gomes VF, Rabna P, et al. Vitamin D as supplementary treatment for tuberculosis: a double-blind, randomized, placebo-controlled trial. Am J Respir Crit Care Med 2009; 179: 843-850.

15 Ralph AP, Waramori G, Pontororing GJ, et al. L-arginine and vitamin D adjunctive therapies in pulmonary tuberculosis: a randomised, double-blind, placebo-controlled trial. PLoS One 2013; 8: e70032.

16 Daley P, Jagannathan V, John KR, et al. Adjunctive vitamin D for treatment of active tuberculosis in India: a randomised, double-blind, placebo-controlled trial. Lancet Infect Dis 2015; 15: 528-534.

17 Tukvadze N, Sanikidze E, Kipiani $\mathrm{M}$, et al. High-dose vitamin $\mathrm{D}_{3}$ in adults with pulmonary tuberculosis: a double-blind randomized controlled trial. Am J Clin Nutr 2015; 102: 1059-1069.

18 Xia J, Shi L, Zhao L, et al. Impact of vitamin D supplementation on the outcome of tuberculosis treatment: a systematic review and meta-analysis of randomized controlled trials. Chin Med J 2014; 127: 3127-3134.

19 Grobler L, Nagpal S, Sudarsanam TD, et al. Nutritional supplements for people being treated for active tuberculosis. Cochrane Database Syst Rev 2016; 11: CD006086.

20 Wallis RS, Zumla A. Vitamin D as adjunctive host-directed therapy in tuberculosis: a systematic review. Open Forum Infect Dis 2016; 3: ofw151.

$21 \mathrm{Wu} \mathrm{HX}$, Xiong XF, Zhu M, et al. Effects of vitamin D supplementation on the outcomes of patients with pulmonary tuberculosis: a systematic review and meta-analysis. BMC Pulm Med 2018; 18: 108.

22 Martineau AR, Jolliffe DA, Hooper RL, et al. Vitamin D supplementation to prevent acute respiratory tract infections: systematic review and meta-analysis of individual participant data. BMJ 2017; 356: i6583.

23 Martineau AR, James WY, Hooper RL, et al. Vitamin $\mathrm{D}_{3}$ supplementation in patients with chronic obstructive pulmonary disease (ViDiCO): a multicentre, double-blind, randomised controlled trial. Lancet Respir Med 2015; 3: $120-130$.

24 Jolliffe DA, Greenberg L, Hooper RL, et al. Vitamin D supplementation to prevent asthma exacerbations: a systematic review and meta-analysis of individual participant data. Lancet Respir Med 2017; 5: 881-890.

25 Jolliffe DA, Greenberg L, Hooper RL, et al. Vitamin D to prevent exacerbations of COPD: systematic review and meta-analysis of individual participant data from randomised controlled trials. Thorax 2019; in press [http://doi. org/10.1136/thoraxjnl-2018-212092].

26 Stewart LA, Clarke M, Rovers M, et al. Preferred reporting items for systematic review and meta-analyses of individual participant data: the PRISMA-IPD statement. JAMA 2015; 313: 1657-1665. 
27 Higgins JP, Altman DG, Gotzsche PC, et al. The Cochrane Collaboration's tool for assessing risk of bias in randomised trials. BMJ 2011; 343: d5928.

28 Phillips PP, Fielding K, Nunn AJ. An evaluation of culture results during treatment for tuberculosis as surrogate endpoints for treatment failure and relapse. PLoS One 2013; 8: e63840.

29 Riley RD, Lambert PC, Abo-Zaid G. Meta-analysis of individual participant data: rationale, conduct, and reporting. $B M J$ 2010; 340: c221.

30 Dept of Health. Nutrition and bone health: with particular reference to calcium and vitamin D. Report Health Soc Subj 1998; 49: 1-24.

31 Rathored J, Sharma SK, Singh B, et al. Risk and outcome of multidrug-resistant tuberculosis: vitamin D receptor polymorphisms and serum 25(OH)D. Int J Tuberc Lung Dis 2012; 16: 1522-1528. 